\title{
RFA vs resection of HCC: exploring the past to improve the future
}

\author{
Marcel C. Langenbach ${ }^{1}$ \\ Received: 11 December 2018 / Revised: 17 December 2018 / Accepted: 4 January 2019 /Published online: 4 February 2019 \\ (C) European Society of Radiology 2019
}

The decision for the treatment of hepatocellular carcinoma (HCC) is based on the Barcelona Cancer Liver Classification (BCLC) which has been established in 1999. The treatment options are still of intense research interest with regular modification of these guidelines.

In general, an individual treatment plan for each patient is evaluated in consent by the multidisciplinary tumor board. In addition to the rigid frame of the BCLC guidelines, there are several factors such as age, comorbidities, and prior treatment which are considered for the final therapy decision [1].

As the BCLC scheme is widely accepted, the several treatment options for all stages of $\mathrm{HCC}$ are undergoing a continuous technical development. Challenging all techniques regarding their feasibility for the different tumor stages is nevertheless important to achieve optimal results for each individual. While patients with low-stage HCC are treated in a curative approach by surgical resection, liver transplantation, or radiofrequency ablation (RFA), patients with advanced-stage HCC can be treated with TACE, sorafenib, or the best supportive care [2].

The authors of the present study retrospectively compared the utilization and effectiveness of RFA and surgical resection of HCC based on the data of the United States National Cancer Database by analyzing more than 18,000 patients from 2004 to 2015 . Several studies already investigated the differences of the two treatment methods for comparable tumor sizes and locations with small study cohorts. In general, the findings were inconclusive across all these studies. Therefore, it is necessary to objectify the outcome of the different treatment options under the consideration of the high variability

This comment refers to the article available at https://doi.org/10.1007/ s00330-018-5902-4.

Marcel C. Langenbach

marcel.langenbach@me.com

1 Institute for Diagnostic and Interventional Radiology, University Hospital Frankfurt, Theodor-Stern-Kai 7, 60590 Frankfurt, Germany across a country with a large-scale population such as the USA. This includes patient- and hospital-related variabilities, which are often difficult to access. The approach of studies based on a national cancer database allows the retrospective proof of therapy strategies for nearly all treatment procedures of several cancer entities. This should be used for a continuous reevaluation of therapy guidelines across all tumor entities and societies. Findings are based on a large variable patient cohort treated in a real-life setting, not in a study setting, and across multiple centers, academic, non-academic, and private practice. However, several limitations which occur in data acquisition for these studies are challenging: first, a therapy standard across the entire study cohort cannot be secured, differences in therapy procedure, especially in interventional treatments, between the several centers, academic and non-academic, are mostly not displayed in a cancer database. Second, registries and databases are often incomplete with no data control by a core institution. And third, there are always patients treated beyond the guidelines as an individual case-based decision.

According to the guidelines, RFA is the appropriate therapy for single lesions $<5 \mathrm{~cm}$ or up to 3 lesions $<3 \mathrm{~cm}$, with several limitations in performance, especially the vicinity to heatsensitive organs. The surgical approach for single lesions $<5 \mathrm{~cm}$ is limited by the local spreading of the tumor, vessel infiltration, or poor liver function. That limits the applicability of both treatment options even if the tumor size is within the guidelines. According to good clinical practice, in patients with an adequate liver function, good localization and sufficient liver reserve, ablation, and resection of tumor lesions $>5 \mathrm{~cm}$ are possible treatment options after a dedicated evaluation of the individual case in a multidisciplinary tumor board [3]. In general, HCC lesions located more in the center of the liver parenchyma are easier to ablate since a surgical approach in these cases may cause a larger parenchymal defect than RFA with a higher risk for liver dysfunction and subsequently worse outcome. In contrast, lesions located close to the liver capsule are easier to resect as the access path is short and the surrounding 
tissue and organs can be spared. RFA shows the best outcome in patients with a single $\mathrm{HCC}$ lesion less than $3 \mathrm{~cm}$ [4].

Based on the results of several smaller studies, RFA has several advantages compared to surgical resection including a shorter duration of hospital stay, better postoperative outcome, lower readmission rates, and better short-time mortality. However, surgical resections show a longer overall survival compared to RFA treatment as the long-term rate of local recurrence is lower. This limits the benefits of RFA. A detailed comparison resulted in a group of elderly patients ( $>65$ years) with small $\mathrm{HCC}$ which showed comparable benefit regarding the overall survival.

There are alternative, less commonly used second- or thirdline treatment options in addition to RFA and surgical resection. For patients with HCC recurrence after RFA, a sequential surgical resection in a curative approach is possible [5]. Further, in patients with a R1 situation after debulking by RFA or resection, an additional treatment with sorafenib has shown promising results [6].

While the therapy strategy for HCC in first-line treatment is well investigated, second- or third-line therapy options in patients with therapy failure, tumor progress, or recurrence are still under intense investigation. Until now, patients with therapy failure are reevaluated and treated like a first-time HCC. For patients with a recurrence, an adjustment of the second- or third-line therapy deviating from the standard pathway is probably more promising. For that reason, therapeutic approaches under recognition of a prior treatment and a probably higher resistance against the therapy should be performed. The combination of several therapy pathways may improve the outcome and survival especially in patients with HCC recurrence. In patients with advanced stage HCC, initial studies of the combination of TACE and sorafenib or the application of VEGF inhibitors with or without the combination with other treatments have shown promising results $[7,8]$. However, these newer treatment alternatives need further investigations to proof a benefit for patients with first-line therapy failure.

In conclusion, the presented large-scale study showed beneficial results of RFA for HCC treatment in the elderly, patients with cirrhosis, and small HCC lesions which is in accordance with prior studies. However, patients who underwent surgical resection had superior overall survival. To extend the validity and include more patient-related details, further investigation in a prospective multicenter setting is necessary. The guidelines as well as the therapy plans should be revised regularly by review of cancer databases and reevaluated as well as readjusted if necessary. In the future, new therapeutic approaches and technically advanced therapy options may result in a better outcome and can probably help to improve the response even after first-line therapy failure.
Funding The author states that this work has not received any funding.

\section{Compliance with ethical standards}

Guarantor The scientific guarantor of this publication is Marcel C. Langenbach.

Conflict of interest The author of this manuscript declares no relationships with any companies, whose products or services may be related to the subject matter of the article.

Statistics and biometry One of the authors has significant statistical expertise.

No complex statistical methods were necessary for this paper.

Informed consent Approval from the institutional animal care committee was not required because the study is an Editorial Comment on a submitted manuscript.

Ethical approval Institutional Review Board approval was not required because an Editorial Comment on a submitted manuscript.

\author{
Methodology \\ - retrospective \\ - observational \\ - performed at one institution
}

Publisher's note Springer Nature remains neutral with regard to jurisdictional claims in published maps and institutional affiliations.

\section{References}

1. Forner A, Reig ME, de Lope CR, Bruix J (2010) Current strategy for staging and treatment: the BCLC update and future prospects. Semin Liver Dis 30:061-074. https://doi.org/10.1055/s-0030-1247133

2. Bruix J, Sherman M, Practice Guidelines Committee, American Association for the Study of Liver Diseases (2005) Management of hepatocellular carcinoma. Hepatology 42:1208-1236. https://doi. org/10.1002/hep.20933

3. Sommer CM, Stampfl U, Kauczor HU, Pereira PL (2014) National S3 guidelines on hepatocellular carcinoma. Radiologe 54:642-653. https://doi.org/10.1007/s00117-014-2656-0

4. Pompili M, Saviano A, de Matthaeis N et al (2013) Long-term effectiveness of resection and radiofrequency ablation for single hepatocellular carcinoma $\leq 3 \mathrm{~cm}$. Results of a multicenter Italian survey. J Hepatol 59:89-97. https://doi.org/10.1016/j.jhep.2013.03.009

5. Yamagishi S, Midorikawa Y, Nakayama H et al (2018) Liver resection for recurrent hepatocellular carcinoma after radiofrequency ablation therapy. Hepatol Res. https://doi.org/10.1111/hepr.13293

6. de Stefano G, Farella N, Scognamiglio U et al (2015) Sorafenib after RFA in HCC patients: a pilot study. Hepatogastroenterology 62:261263

7. Geschwind JF, Kudo M, Marrero JA et al (2016) TACE treatment in patients with Sorafenib-treated Unresectable hepatocellular carcinoma in clinical practice: final analysis of GIDEON. Radiology 279: 630-640. https://doi.org/10.1148/radiol.2015150667

8. Longo V, Gnoni A, Casadei Gardini A et al (2017) Immunotherapeutic approaches for hepatocellular carcinoma. Oncotarget 8:33897-33910. https://doi.org/10.18632/oncotarget. 15406 